\title{
Cognitive remediation therapy for patients with bipolar disorder: A randomised proof-of-concept trial
}

\author{
Rebecca Strawbridge $^{1}$ (D) | Dimosthenis Tsapekos ${ }^{1}$ (D) | John Hodsoll ${ }^{2}$ (D) | \\ Tim Mantingh ${ }^{1}$ (D) | Nefize Yalin ${ }^{1}$ (D) | Paul McCrone ${ }^{3}$ (D) | Janet Boadu ${ }^{3}$ (D) | \\ Karine Macritchie $^{4}$ (D) | Matteo Cella ${ }^{5}$ (D) $\mid$ Clare Reeder $^{5}$ (D) | Jessica Fish ${ }^{5}$ (D) | \\ Til Wykes $^{5}$ (D) | Allan H. Young ${ }^{1,4}$ (D)
}

\begin{abstract}
${ }^{1}$ Department of Psychological Medicine, Institute of Psychiatry, Psychology \& Neuroscience, King's College London, London, UK

${ }^{2}$ Department of Biostatistics, King's Clinical Trials Unit, King's College London, London, UK

${ }^{3}$ Department of Health Services and Population Research, King's Health Economics, Institute of Psychiatry, Psychology \& Neuroscience, King's College London, London, UK

${ }^{4}$ OPTIMA Mood Disorders Service, Lambeth Hospital, South London and Maudsley NHS Foundation Trust, London, UK

${ }^{5}$ Department of Psychology, Institute of Psychiatry, Psychology \& Neuroscience, King's College London, London, UK
\end{abstract}

\section{Correspondence}

Allan H. Young, Department of Psychological Medicine PO72, Institute of Psychiatry, Psychology \& Neuroscience, King's College London, London, SE5 8AF, UK.

Email: Allan.young@kcl.ac.uk

Funding information

National Institute of Health Research (NIHR), Grant/Award Number: ID PB-PG-0614-34075; NIHR Maudsley Biomedical Research Centre

\begin{abstract}
Objectives: Cognitive remediation therapy (CRT) may benefit people with bipolar disorder type I and II for whom cognitive impairment is a major contributor to disability. Extensive research has demonstrated CRT to improve cognition and psychosocial functioning in people with different diagnoses, but randomised trials of evidenced therapy programmes are lacking for bipolar disorders. The Cognitive Remediation in Bipolar (CRiB) study aimed to determine whether an established CRT programme is feasible and acceptable for people with bipolar disorders.

Methods: This proof-of-concept, single-blind randomised trial recruited participants aged 18-65 with bipolar disorder, not currently experiencing an episode. They were 1:1 block randomised to treatment-as-usual (TAU) with or without individual CRT for 12 weeks. The partly computerised CRT programme ("CIRCuiTS") was therapist-led and is evidence-based from trials in those with psychotic illnesses. Data were collected and analysed by investigators blinded to group allocation. The main outcomes (week 13 and 25) examined participant retention, intervention feasibility and putative effects of CRT on cognitive and psychosocial functioning via intention-to-treat analyses. Trial registration: ISRCTN ID32290525.

Results: Sixty participants were recruited (02/2016-06/2018) and randomised to CRT $(n=29)$ or TAU $(n=31)$. Trial withdrawals were equivalent (CRT $n=2 / 29$; TAU $n=5 / 31)$. CRT satisfaction indicated high acceptability. Intention-to-treat analyses $(\mathrm{N}=60)$ demonstrated greater improvements for CRT- than TAU-randomised participants: at both week 13 and 25, CIRCuiTS participants showed larger improvements in the following domains (week 25 effect sizes reported here): IQ (SES $=0.71,95 \%$ $\mathrm{Cl}[0.29,1.13])$, working memory (SES $=0.70,95 \% \mathrm{Cl}[0.31,1.10]$ ), executive function (SES $=0.93,95 \% \mathrm{Cl}[0.33,1.54]$ ), psychosocial functioning (SES $=0.49,95 \% \mathrm{Cl}$ $[0.18,0.80]$ ) and goal attainment $(\mathrm{SES}=2.02,95 \% \mathrm{Cl}[0.89,3.14])$. No serious adverse events were reported.
\end{abstract}


Conclusions: CRT is feasible for individuals with bipolar disorders and may enhance cognition and functioning. The reported effect sizes from this proof-of-concept trial encourage further investigation in a definitive trial.

KEYWORDS

bipolar, cognitive enhancement, cognitive remediation, CRT, randomised trial

\section{1 | INTRODUCTION}

Bipolar disorder (BD) is common, complex, and often inadequately treated: calls have begun to pursue evidence-based therapies that target fundamental cognitive process ${ }^{1}$ since two thirds of patients suffer cognitive deficits which are often pronounced and widespread (spanning memory, attention, and executive functions), persisting throughout periods of recovery and directly linked to psychosocial and occupational functioning impairments. ${ }^{2}$ As well as functional impacts, a pattern of cognitive deterioration may increase episode recurrence, so improving cognitive abilities might enhance not only short-term wellbeing but potentially the future course of the disorder. ${ }^{1,3,4}$ Cognitive Remediation Therapy (CRT) is a psychological intervention that has demonstrated reliable benefits for people with schizophrenia, improving core cognitive functioning, daily functioning, and quality-of-life as determined through extensive examination via clinical trials (efficacy, effectiveness, mechanistic) and comprehensive meta-analytic syntheses. ${ }^{\text {eg5-7 }}$ There are well-documented similarities between the cognitive difficulties experienced by people with schizophrenia and bipolar disorder: they appear comparable in terms of impact and quality, although are on average less severe for patients with BD than schizophrenia and there is less evidence of a premorbid component. ${ }^{8}$ Preliminary evidence for the potential benefits of CRT for BD is encouraging: an initial meta-analysis found analogous cognitive effects in trials including some participants with affective disorders ( $E S=0.44$ ) compared to those only assessing individuals with psychotic disorders $(E S=0.36) .{ }^{7}$ A handful of small or non-randomised studies have ensued, recently synthesised in systematic reviews ${ }^{3,4}$ reporting only two published randomised trials of CRT (one for those with psychotic bipolar disorder, ${ }^{9}$ the other employing a short-term group therapy paradigm for a small sample of patients with $\mathrm{BD}^{10}$ ) concluding that individual CRT provided more intensively or for a longer duration might demonstrate meaningful benefits.

We present here the findings of a randomised proof-of-concept trial (the "CRiB" study) comparing an evidence-derived, intensive, individual manualised CRT intervention with treatment-as-usual. The $\mathrm{CRiB}$ trial aimed to determine the potential for CRT development into a new evidence-based therapy for bipolar disorder (type I and II) by investigating four primary outcomes: whether an established therapist-led CRT programme is 1) feasible and 2) acceptable for this patient group and whether it yields benefits in comparison to usual care, in terms of 3) improved cognitive performance and 4) improved psychosocial functioning.

\section{MATERIALS AND METHODS}

\section{1 | Study design}

This was a single-blind, proof-of-concept randomised trial conducted in one UK centre within an academic clinical research facility (South London \& Maudsley NHS Trust and King's College London). The trial protocol contains full details of the study methodology. ${ }^{11}$ The authors assert that all procedures contributing to this work comply with the ethical standards of the relevant national and institutional committees on human experimentation and with the Helsinki Declaration of 1975 , as revised in 2008. All procedures involving human subjects/patients were approved by City Road \& Hampstead UK Research Ethics Committee (16/10/2015, reference 15/LO/1557).

\section{2 | Participants}

Participants were eligible if they had a diagnosis of BD, were currently euthymic, aged 18-65 years without meeting exclusion criteria (not being able to communicate in English, not being able to use a computer, have a substantial neurological illness, substanceuse, or personality disorder). No other eligibility restrictions were placed on somatic illnesses or medications taken either upon trial entry or medication changes throughout participation, in line with usual care. However, we did ask patients whether they were undergoing a psychological therapy at present or planning any treatment changes in the near future and if so, delayed screening for study eligibility. Current euthymia was defined as scoring $<8$ on the Hamilton Rating Scale for Depression (HAMD) ${ }^{12}$ and Young Mania Rating Scale (YMRS) ${ }^{13}$ on two occasions one week apart assessing mood over the preceding month. Eligibility, including diagnosis, was assessed by trained researchers (DT, TM, RS) and validated by a psychiatrist (NY, KM, AHY). Participants were recruited via secondary and primary care services as well as off- and on-line advertisement from the community. Written informed consent was obtained from all individuals before participating, following full explanations of the procedures involved.

\section{3 | Randomisation and masking}

Participants were assigned to receive CRT in addition to treatmentas-usual (TAU) or TAU alone following completion of the baseline 
assessment (week 0). A 1:1 block allocation design with random and variable block size was generated by an independent, validated web-based randomisation system including concealed sequence allocation and locked randomisation details after allocation, as detailed in the protocol. ${ }^{11}$ Randomisation was stratified by the intensity of TAU as assessed by the Client Service Receipt Inventory (CSRI) service-use assessment ${ }^{14}$ (intensive or non-intensive TAU, defined respectively as more or less than monthly appointments with a healthcare professional). Investigators responsible for obtaining informed consent and undertaking data collection (including symptoms, functioning, and cognitive outcome ratings; DT/ TM) were blinded to group allocation; the trial statistician (JH) was also blinded. To maintain investigator blinding, the unblinded trial coordinator (RS) carried out randomisation, conveyed allocation by telephone or in person to therapists and participants, requesting for participants to not disclose their group allocation to data collectors. Those delivering and receiving CRT intervention were unblinded out of necessity.

\section{4 | Intervention}

Over 12 weeks, CRT was delivered by trained postgraduate psychologists with supervision from clinical psychologists with $>5$ years CRT experience. The metacognition-informed, therapist-led, computerised CRT program "CIRCuiTS" emphasises strategy use and transfer having been established in populations diagnosed with psychoses ${ }^{6,15,16}$; In addition to compensatory (or strategy-based) remediation approaches, all session formats also included a restorative remediation element (eg, "drill and practice" of cognitive tasks that were clearly distinct from cognitive outcome assessments). CRT flexibly utilised a combination of formats (in-person, telephone, and individual practice sessions) which were arranged under the responsibility of the therapist, accommodating the patients' needs. Target engagement comprised three sessions per week including at least one session guided by the therapist each week, with a target total of 20-30 hours. The minimum dose of 20 hours was proposed following evidence in schizophrenia research. ${ }^{16}$ Appendix A contains additional details.

\section{$2.5 \mid$ Procedures}

As detailed in the protocol, ${ }^{11}$ all participants continued receiving usual care throughout the trial. After baseline assessment and randomisation (week 0), the 12-week intervention period comprised of CRT + TAU or TAU alone. After the intervention period, all outcomes (cognitive, functional, acceptability) were repeated at a post-intervention assessment (week 13) and a follow-up assessment 12 weeks later (week 25). ${ }^{11}$ Each assessment was conducted in a private, quiet room and was only undertaken where participants had capacity to consent and complete the assessments. Participants were instructed to sleep well (as much as possible) the night before assessments and to entirely avoid use of alcohol or non-prescribed drugs on the day of assessments. Cognitive tests were undertaken as close as possible to 1-4 PM to standardise daily fluctuations in alertness.

\section{6 | Outcomes}

As detailed in the protocol, the four main feasibility outcomes were considered primary with equal weighting. These, and the measures used to examine them, were:

1. Trial feasibility: recruitment (number consenting to participate) and completion (number completing the final outcome assessment) rates assessed acceptability of randomisation to TAU relative to CRT. No specific criteria were set a priori to determine sufficient feasibility (recruitment/ completion rates) to progress the research to a comprehensive future trial.

2. CRT intervention acceptability: the number of participants completing 20 hours of CRT, average number of hours completed, and CRT satisfaction questionnaire ratings. ${ }^{17}$ Time spent in sessions with a therapist and independent CIRCuiTS practice were summed to calculate completion time. Service use and healthrelated quality-of-life were assessed for health economic analyses (for details, see Statistical Analysis section below). ${ }^{14,18}$

3. Cognitive outcomes: the cognition battery measured processing speed, attention (digit symbol substitution test [DSST], symbol search test [SS]) and working memory (digit span [DS]), ${ }^{19}$ verbal learning (verbal paired associates I [VPA1]) and memory (verbal paired associates II [VPA2]), ${ }^{20}$ current IQ [WASI], ${ }^{21}$ verbal fluency $[F A S],{ }^{22}$ executive function [Hotel test] ${ }^{23}$ and perceived cognitive impairment (perceived deficits questionnaire [PDQ]). ${ }^{24} \mathrm{~A}$ composite score of cognitive function (processing speed, working memory, verbal memory, verbal fluency, IQ, executive function domains) was calculated to examine global cognition. ${ }^{11}$ Of these, the DSST was proposed as a potential primary outcome for a future efficacy CRT trial because it has shown high sensitivity to change over time. ${ }^{19}$ Although IQ is commonly considered a stable trait the WASI assessment has been validated to show variability over time and is commonly used as a "current intellectual functioning" tool. ${ }^{21}$ The cognitive tests and the UPSA (below) have known practice effects, thus it was expected that improvements would be observed over time across the sample and betweengroups comparisons addressed this to indicate relative performance between those randomised to TAU vs CRT.

4. Functional outcomes: functional capacity (UCSD performancebased skills assessment [UPSA]), ${ }^{25}$ psychosocial functioning (functioning assessment short test [FAST] ${ }^{26}$ and achievement of patient-defined goals (goal attainment scale[GAS]). ${ }^{27}$ FAST utilises a semi-structured interview to rate level of functioning in the domains of autonomy, financial, interpersonal, leisure, cognitive and occupational functioning (denoted forthwith as "psychosocial functioning"). 
Additionally, symptoms of depression ${ }^{12}$ and mania $^{13}$ were monitored and adverse events recorded throughout.

\subsection{Statistical analysis}

The target sample size of 60 was selected due to recommendations for pilot trials to estimate efficacy. ${ }^{28}$ All planned analyses adopted the intention-to-treat (ITT) approach. As detailed in the protocol ${ }^{11}$ descriptive statistics summarized participant characteristics as appropriate (mean, standard deviation [SD], median, interquartile range [IQR], frequency and percentage [\%]). Inferential statistics produced group differences and standardised effect sizes (SES) with confidence intervals $\left(95 \% \mathrm{Cl}\right.$ ) computed using R version 3.6.1. ${ }^{29}$

\subsection{1 | Outcome 1-Trial feasibility}

Descriptively, the rate of consenting eligible participants, assessment non-completion and study withdrawals were examined alongside reasons for each trial arm.

\subsection{2 | Outcome 2-CRT intervention acceptability}

Descriptive statistics examined intervention acceptability (CRT satisfaction questionnaire) ${ }^{17}$ and feasibility (number of participants completing 20 therapy sessions, total hours engaged with therapy, reasons for non-engagement). A health and social care perspective was adopted in the cost-effectiveness. The economic costs of CRT and TAU services were calculated; service-use during the trial was measured by the CSRI ${ }^{14}$ and combined with standard unit costs of these services ${ }^{30}$ added to intervention costs (the resources required to deliver CIRCuiTS, eg, therapist time, overheads). These were combined with quality-adjusted life years derived from the EQ-5D-3L ${ }^{18}$ using area under the curve methods to compare groups. ${ }^{11}$

\subsection{3 | Outcomes 3 and 4-putative effects of CRT on cognition and function}

Intervention effect sizes (ES) were estimated using linear mixed models for each outcome measurement at both post-treatment (week 13) and follow-up (week 25) with outcome timepoint as dependent variable plus fixed effects of trial arm (CRT vs TAU) and baseline measures of outcome. Standardised effect sizes (SES) and $95 \% \mathrm{Cl}$ were calculated by dividing the group mean differences by standard deviation of the baseline outcome score. The following convention was used to interpret effect size: small 0.2-0.49, moderate 0.5-0.79, large $\geq 0.8$. As a pilot study, estimation is focused on ES but includes $p$ values as an aid to interpretation. Missing data were dealt with using a maximum likelihood (ML) approach with the missing at random
(MAR) assumption. Baseline predictors of missingness were sought, to be included in the analysis model if an association was found at a liberal $P<.2$ criterion. The study was monitored by a Trial Steering Committee and the trial was pre-registered in the ISRCTN registry (ID32290525; http://www.isrctn.com/ISRCTN32290525).

\subsection{Changes to the protocol}

Compared to the published protocol, minor changes since trial commencement are as follows:

1. Diagnostic subtype: only patients with bipolar disorder type I were eligible. From November 2016 (recruitment month 9, after $\mathrm{n}=25$ ), eligibility was broadened to include individuals with BD type II in response to lack of evidence suggesting cognitive differences between diagnostic subtypes. ${ }^{2}$

2. Sub-analyses: We had planned to analyse differences in cognition over the menstrual cycle but many patients were not currently menstruating and therefore these analyses were not included.

3. Functional capacity: A practical test of functioning was added to the outcome assessment protocol prior to trial commencement, to assess function using an applied measure. The UPSA ${ }^{25}$ is a recommended outcome measure in cognitive trials due to its moderate correlations with cognitive performance and psychosocial functioning. ${ }^{31}$

\section{3 | RESULTS}

Between 02/2016 and 06/2018, 60 participants were recruited and randomised (CRT $n=29$, TAU $n=31$ ) and included in analysis. The CONSORT diagram delineates the flow of participants throughout the trial (Figure 1).

Participant characteristics (Table 1) indicate overall comparability between CRT and TAU groups. The sample consisted mostly of women (68\%; CRT 72\%, TAU 65\%) with a median age of 42 (IQR; 31-52). Most participants met diagnostic criteria for type I BD (70\%; CRT 69\%, TAU 71\%) and the remaining for type II. Participants were taking a median of two psychotropic medications, most commonly antipsychotics and approximately half the sample reported a physical health condition and were taking non-psychotropic medication. Clinical characteristics are displayed in Appendix Table A1.

\section{4 | TRIAL FEASIBILITY}

\subsection{Recruitment rate}

A mean of 2.3 participants per month were recruited and consented into the trial ( $S D=0.97$, range $0-5$ ). For a sample size of 60,226 potential participants were identified, giving a recruitment rate of $36 \%$ 


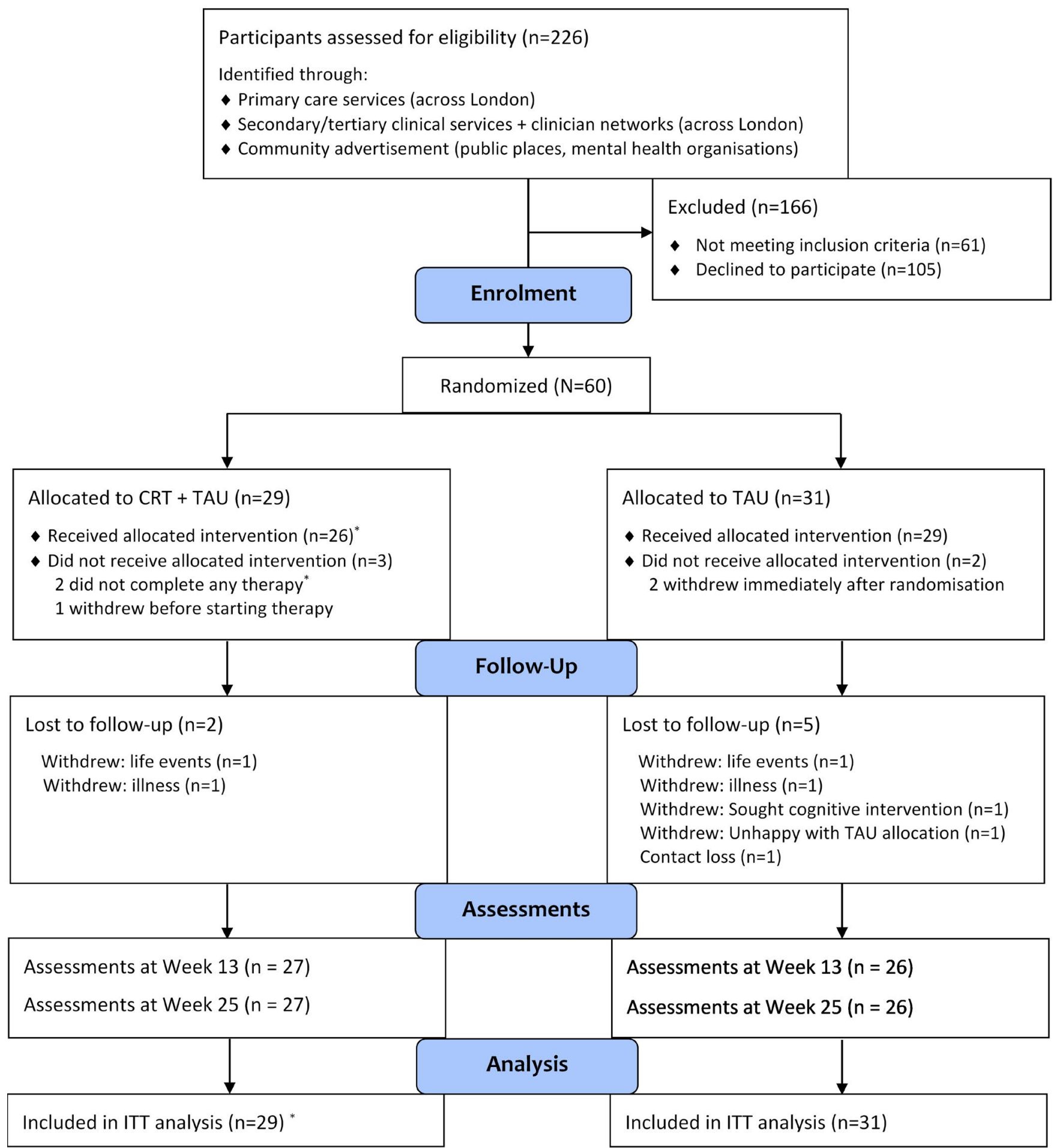

FIGURE 1 CONSORT diagram. *26 participants received at least some CRT therapy. The two participants who did not complete any therapy plus two who completed $<10$ hours CRT had the same therapist who did not engage with the study. One further participant was identified subsequently as meeting an exclusion criterion for the study (10-19 hours therapy). These five individuals were subject to sensitivity analysis for cognitive and functional outcomes because they were considered to not have been treated in full adherence to the protocol, unlike other participants (including those who withdrew, who were not excluded in per-protocol analyses)

(95\% Cl [29\%, 44\%]). The most common reason for non-participation was inability to commit to appointments at the research site multiple times per week for 12 weeks for CRT, most frequently due to long travel distances required to attend.

\subsection{Completion rate}

Of the 60 individuals randomised, seven participants (12\%, 95\% $\mathrm{Cl}[5 \%, 22 \%])$ were lost to follow-up due to withdrawal $(\mathrm{n}=6)$ or 
TAB LE 1 Participant characteristics at baseline

\begin{tabular}{|c|c|c|c|c|}
\hline Variable & Labels & $\operatorname{TAU}(n=31)$ & CRT $(n=29)$ & $\begin{array}{l}\text { All } \\
(N=60)\end{array}$ \\
\hline Age & med (IQR) & $42.5(31.8,52.2)$ & $43(34,52.5)$ & $42(31,52)$ \\
\hline$G$ ender ${ }^{a}$ & Female $\mathrm{n}(\%)$ & $20(64.5)$ & $21(72.4)$ & $41(68.3)$ \\
\hline \multirow[t]{4}{*}{ Ethnicity } & White British n (\%) & $21(67.7)$ & $17(58.6)$ & $38(63.3)$ \\
\hline & White Other n (\%) & $5(16.1)$ & $7(24.1)$ & $12(20)$ \\
\hline & $\begin{array}{l}\text { Non-White Other } \\
\mathrm{n}(\%)\end{array}$ & $3(9.7)$ & $1(3.4)$ & $4(6.7)$ \\
\hline & Mixed n (\%) & $0(0)$ & $1(3.4)$ & $1(1.7)$ \\
\hline Education (yrs) & med (IQR) & $16(15,17)$ & $16(15,17)$ & $16(14,18)$ \\
\hline BMI & med(IQR) & $28(26,32)$ & $27(24,30)$ & $27(25,31)$ \\
\hline Physical health condition & n (\%) & $13(42)$ & $18(62)$ & $31(52)$ \\
\hline \multirow[t]{2}{*}{ Bipolar diagnosis } & Type I n (\%) & $22(71)$ & $20(69)$ & $42(70)$ \\
\hline & Type II n (\%) & 9 (29) & $9(31)$ & $18(30)$ \\
\hline \multirow[t]{3}{*}{ Current psychotropic medications } & $\begin{array}{l}\text { Total number med } \\
\text { (IQR) }\end{array}$ & $2(2,3)$ & $2(2,3)$ & $2(2,3)$ \\
\hline & Lithium n (\%) & $14(45.2)$ & $7(24.1)$ & $21(35)$ \\
\hline & Anticonvulsants $\mathrm{n}(\%)$ & $18(58.1)$ & $17(58.6)$ & $35(58.3)$ \\
\hline Total number of episodes & med (IQR) & $13.5(8,24.2)$ & $13(8,20.5)$ & $15(8,30)$ \\
\hline Depression severity (HAMD) & med (IQR) & $3(1,5.5)$ & $4(3,7)$ & $4(2,6)$ \\
\hline Mania severity (YMRS) & med (IQR) & $1(0,3)$ & $2(1,4)$ & $1(0,4)$ \\
\hline
\end{tabular}

Abbreviations: BMI, body mass index; CRT, cognitive remediation therapy; HAMD, Hamilton rating scale for depression 17-item version; IQR, interquartile range; med, median; TAU, treatment as usual; YMRS, Young mania rating scale.

${ }^{a}$ Gender was determined by asking patients their gender (as an open-ended question); all responded as male/man or female/woman.

contact loss $(n=1)$. The odds of dropout were non-significantly higher in TAU $(n=5)$ than CRT $(n=2)$ groups; OR $=2.31, P=.44$. Withdrawal immediately after randomization occurred for one CRT participant (life events) and two TAU participants (one unsatisfied with randomization to TAU; one life events). Loss at follow-up during the intervention period occurred for one CRT participant (illness) and three TAU participants (one sought other cognitive intervention; one illness; one contact loss). All other participants attended all assessments: unless specified, all analyses include all participants. No demographic or clinical baseline predictors of missing data were identified (all $P>$.25).

\section{3 | Intervention feasibility}

\subsection{1 | CRT completion}

$22 / 29$ participants completed $>20$ hours of therapy (76\%). Including all 29 participants, a mean of 25 (SD $=13$, range $0-48$ ) CRT hours were completed. The 22 completers undertook a mean of 31 hours (SD $=7$, range 22-48). Of the seven non-completers, two withdrew from the trial (described above), one was considered to have a substance-use disorder (deviation from protocol) during CRT which impeded engagement (10-19 therapy hours). The other four 
participants were allocated the same therapist: this therapist was subsequently found to not comply with study protocols (including adherence to treatment guidelines and timely communications with participants), which was thus considered a protocol deviation. Of these four participants, two did not begin therapy and two completed $<10$ hours. These five participants whose participation in the trial were considered to deviate from the original protocol were subject to sensitivity analysis ( $\mathrm{N}=55$ is referred to as "per-protocol," $\mathrm{N}=60$ as "ITT analysis").

\subsection{2 | CRT satisfaction}

The satisfaction questionnaire $(n=21)$ indicated acceptability (Appendix Table A2); 95\% said they improved after CRT, 86\% felt there were the right number of sessions and $100 \%$ liked their therapist. $76 \%$ agreed that CRT made them more aware of their limitations; all rated increased awareness as helpful rather than frustrating. Health economics: Service use was similar between groups although treatment costs were non-significantly higher for those receiving TAU. This was the case before accounting for CRT costs (week 0: TAU $£ 1,880$ and CRT + TAU £1,744 (£135 higher, 95\% Cl [-£1873, 1221]); week 13, TAU £809, CRT + TAU £794 ( $£ 16$ higher, 95\% Cl [-£498, 459]), week 25 TAU £649, CRT + TAU £618 (£32 higher, 95\% Cl [-£314, 251]) and after CRT costs ( $£ 629.70$ per participant) were added to usual care during follow-up (CRT £2082, TAU $£ 1458 ; 95 \% \mathrm{Cl}$ difference $[-£ 80, £ 1238])$. Health-related qualityof-life improved slightly between weeks 0 and 13 similarly between groups. QALY values accrued over the follow-up period were similar $(C R T+$ TAU mean $=0.40, S D=0.09 ;$ TAU mean $=0.41 ; \mathrm{SD}=0.07)$; with little difference after controlling for baseline $(-0.002 ; 95 \% \mathrm{Cl}$ $[-0.035,0.032])$. Overall, CRT + TAU resulted in non-significantly higher overall costs and slightly fewer QALYs and was therefore "dominated" by TAU alone. However, there was much uncertainty around the estimates.

\section{4 | Putative cognitive effects of CRT}

Table 2 displays cognitive and functioning scores throughout the trial. At baseline, 40 (67\%) of patients scored $\geq 0.5$ SD below the normative mean in two or more individual cognitive tests; the proportion of patients impaired according to recommended definitions ${ }^{31}$ are presented in Table 2 alongside raw scores. Cognitive outcome analyses are displayed in Table 3: Both groups improved between baseline and subsequent assessments. Both mean difference effect sizes after treatment (see also Appendix Table A3), and linear mixed models (Table 3), demonstrated greater improvements in the CRT than TAU groups. Linear mixed models indicated that CRT participants showed larger improvements than TAU participants at both posttreatment (week 13) and follow-up (week 25) for IQ (WASI: week 13 $\mathrm{SES}=0.52,95 \% \mathrm{Cl}[0.10,0.94], P=.015$; week $25 \mathrm{SES}=0.71,95 \%$ $\mathrm{Cl}[0.29,1.13], P=.001)$, working memory (DS: week $13 \mathrm{SES}=0.45$,
95\% Cl [0.06, 0.84], $P=.024$; week $25 \mathrm{SES}=0.70,95 \% \mathrm{Cl}[0.31$, $1.10], P=.001$ ) and executive function (Hotel: week $13 \mathrm{SES}=0.68$, $95 \% \mathrm{Cl}[0.08,1.28], P=.027$; week $25 \mathrm{SES}=0.93,95 \% \mathrm{Cl}[0.33$, 1.54], $P=.003$ ). Additionally, this effect was observed at week 25 for global cognition (composite test: SES $=0.6895 \% \mathrm{Cl}[0.28$, 1.06], $P=.001$ ), memory recall (VPA2: $\mathrm{SES}=0.45,95 \% \mathrm{Cl}[0.13$, $0.78], P=.007$ ), verbal fluency (FAS: SES $=0.52,95 \% \mathrm{Cl}[0.17,0.86]$, $P=.004)$ and processing speed (DSST: SES $=0.35,95 \% \mathrm{Cl}[0.03$, 0.67], $P=.031$ and SS: SES $=0.66,95 \% \mathrm{Cl}[0.25,1.06], P=.002)$. Figure 2 depicts cognitive changes between groups as analysed by linear mixed models.

\section{5 | Putative functional effects of CRT}

At baseline, 27 (55\%) of participants were categorised as having moderate to severe impairments in functioning while 33 (45\%) had no or mild functional impairment, according to validated FAST cut-off scores ${ }^{32}$ (see Table 2). Similar to cognition, between-group effect sizes indicate larger improvements in CRT than TAU participants on functional measures (Table 2). Linear mixed models (see Table 3) identified significantly greater improvements at weeks 13 and 25 for psychosocial functioning (FAST: week 13 SES $=0.45$, $95 \% \mathrm{Cl}[0.15,0.76], P=.004$; week $25 \mathrm{SES}=0.49,95 \% \mathrm{Cl}[0.18$, $0.80], P=.002$ ) and goal attainment (GAS: week $13 \mathrm{SES}=3.46,95 \%$ $\mathrm{Cl}[2.33,4.58], P<.001$; week $25 \mathrm{SES}=2.02,95 \% \mathrm{Cl}[0.89,3.14]$, $P=.001)$. At week 13 but not 25 , functional capacity was more improved in CRT participants (UPSA: SES $=0.56,95 \% \mathrm{Cl}[0.11,1.01]$, $P=.015)$. Sensitivity analyses demonstrated consistently greater benefits for per-protocol CRT than TAU participants $(n=55)$ across cognition and functioning at both post-treatment timepoints; see Appendix Table A4.

\subsection{1 | Affective symptoms}

Symptoms were similar between groups (see Appendix Table A1), although participants in the CRT group had slightly higher subsyndromal depression (HAM-D) scores at week 25 (TAU mean $=4$, $\mathrm{SD}=3 ; \mathrm{CRT}$ mean $=6, \mathrm{SD}=4 ; \mathrm{ES}=0.59,95 \% \mathrm{Cl}[0.03,1.15])$. No full relapses were recorded in participants attending follow-up visits; four participants at week 25 had mild depressive symptoms (HAMD score 14-15; 3 CRT, 1 TAU) and one participant had mild mania symptoms (YMRS 20; TAU participant).

\subsection{2 | Adverse events}

No serious adverse events occurred. Four adverse events were recorded, each for different participants, related to study procedures (experience of distress at feeling they performed poorly on cognitive tests, $n=2$ ) or bipolar illness (depressive episode, $n=2$ ). No suicide attempts or self-harm were disclosed to the study team. 
TAB LE 2 Cognitive and functional measures at all time points

\begin{tabular}{|c|c|c|c|c|c|}
\hline Test & $\mathrm{N}(\%) \geq 0.5 \mathrm{SD}$ below mean ${ }^{\mathrm{a}}$ & Arm & $\frac{\text { Baseline (Week 0) }}{\text { Median (IQR) }}$ & $\begin{array}{l}\text { Post-treatment (Week 13) } \\
\text { Median (IQR) }\end{array}$ & $\frac{\text { Follow-up (Week 25) }}{\text { Median (IQR) }}$ \\
\hline \multirow[t]{3}{*}{ DSST } & \multirow[t]{3}{*}{$24(40 \%)$} & All & $62(53.75,75.25)$ & $64(59,78)$ & $69(59,79)$ \\
\hline & & TAU & $61(53.5,73.5)$ & $63.5(56.5,76.25)$ & $65.5(58,77.5)$ \\
\hline & & CRT & $63(54,76)$ & $67(60,79)$ & $74(60,85)$ \\
\hline \multirow[t]{2}{*}{ WASI } & \multirow[t]{2}{*}{$12(20 \%)$} & All & $109(97.5,113.5)$ & $109(101,119)$ & $112(101,118)$ \\
\hline & & CRT & $111(104,117)$ & $117(106.5,122)$ & $116(109.5,124)$ \\
\hline \multirow[t]{3}{*}{ VPA1 } & \multirow[t]{3}{*}{$25(42 \%)$} & All & $29(20.75,39.25)$ & $33(22,47)$ & $41(30,50)$ \\
\hline & & TAU & $25(17.5,34.5)$ & $30(22,47.5)$ & $33(25.5,45)$ \\
\hline & & CRT & $36(26,43)$ & $38(26.5,47)$ & $49(35.5,52)$ \\
\hline \multirow[t]{3}{*}{ VPA2 recognition } & \multirow[t]{3}{*}{$20(33 \%)$} & All & $39(36,40)$ & $40(38,40)$ & $40(39,40)$ \\
\hline & & TAU & $38(35,40)$ & $39(37,40)$ & $39(37.3,40)$ \\
\hline & & CRT & $39(37,40)$ & $40(38.5,40)$ & $40(39,40)$ \\
\hline \multirow[t]{3}{*}{ DS } & \multirow[t]{3}{*}{9 (15\%) } & All & $27(25.75,32)$ & $29(27,32)$ & $30(27,32)$ \\
\hline & & TAU & $30(25,33)$ & $29.5(26.25,32)$ & $29(26,31)$ \\
\hline & & CRT & $27(26,32)$ & $29(27,32.5)$ & $31(28,35.5)$ \\
\hline \multirow[t]{3}{*}{ SS } & \multirow[t]{3}{*}{$18(30 \%)$} & All & $32(27,35.25)$ & $34(28,39)$ & $33(27,40)$ \\
\hline & & TAU & $31(28,34.5)$ & $33.5(26.5,37.75)$ & $32(25.75,36.5)$ \\
\hline & & CRT & $33(26,37)$ & $34(29.5,39)$ & $37(28,41.5)$ \\
\hline Global & 22 (37\%) & All & $2.8(2.51,3.26)$ & $3.2(2.71,3.51)$ & $3.2(2.83,3.67)$ \\
\hline & & TAU & $2.8(2.35,3.14)$ & $3.0(2.60,3.32)$ & $3.1(2.69,3.38)$ \\
\hline & & CRT & $3.00(2.58,3.32)$ & $3.2(2.89,3.72)$ & $3.6(3.06,4.00)$ \\
\hline PDQ & $\mathrm{n} / \mathrm{a}$ & All & $36(27,44)$ & $30(20,37)$ & $30(19,39)$ \\
\hline & & TAU & $35(25.5,44.5)$ & $29.5(17,41)$ & $31(12.75,37.75)$ \\
\hline & & CRT & $37(28,43)$ & $31(21,36.5)$ & $30(20.5,40.5)$ \\
\hline UPSA & $\mathrm{n} / \mathrm{a}$ & All & $74(67,81.75)$ & $81(72,86)$ & $84(74,93)$ \\
\hline & & TAU & $71.5(65.75,82.25)$ & $78(68,86)$ & $80(74,88)$ \\
\hline & & CRT & $74(69.75,81.75)$ & $81(79,86)$ & $86(81,93)$ \\
\hline FAST & $\mathrm{n} / \mathrm{a}$ & All & $21.5(13,29.25)$ & $20(11,29)$ & $22(11,28)$ \\
\hline & & TAU & $19(12.5,26)$ & $19(13.25,26.75)$ & $21.5(13,26.5)$ \\
\hline & & CRT & $25(15,32)$ & $22(10,29)$ & $22(10.5,29)$ \\
\hline GAS & $\mathrm{n} / \mathrm{a}$ & All & $36.4(31.13,37.6)$ & $44.9(38.8,52.05)$ & $45.9(39.65,54.9)$ \\
\hline & & TAU & $36.5(31.15,37.6)$ & $39.9(32.4,44.2)$ & $42.2(36.8,47.3)$ \\
\hline & & CRT & $34.6(30.97,37.6)$ & $51.2(45.17,60.53)$ & $51.5(43.95,57.1)$ \\
\hline
\end{tabular}

Bold values are used to highlight the rows which contain all participants, those not in bold are the subsamples split by randomisation group. Abbreviations: DS, Digit Span; DSST, Digit Symbol Substitution Test; FAS, verbal fluency test; FAST, Functioning Assessment Short Test; GAS, goal attainment scale; PDQ, perceived deficits questionnaire; SS, Symbol Search; UPSA, UCSD Performance-based Skills Assessment; VPA, Verbal Paired Associates; WASI, IQ.

aproportion impaired compared to demographically-corrected normative values. All other cognitive data are presented as raw scores. 
TABLE 3 Cognitive and functional outcomes between CRT and TAU (time $x$ group interactions)

\begin{tabular}{|c|c|c|c|c|c|c|c|}
\hline & Measure & \multicolumn{3}{|l|}{ Week 13} & \multicolumn{3}{|l|}{ Week 25} \\
\hline \multirow{2}{*}{$\begin{array}{l}\text { Processing speed/ } \\
\text { attention }\end{array}$} & DSST & $0.25(-0.07,0.57)$ & $1.57(69.8)$ & 0.122 & $0.35(0.03,0.67)$ & $2.2(69.8)$ & 0.031 \\
\hline & SS & $0.26(-0.15,0.67)$ & $1.25(93.5)$ & 0.213 & $0.66(0.25,1.06)$ & $3.19(93.5)$ & 0.002 \\
\hline Working memory & DS & $0.45(0.06,0.84)$ & $2.3(78.1)$ & 0.024 & $0.7(0.31,1.1)$ & $3.59(78.1)$ & 0.001 \\
\hline Verbal memory & Recognition & $0.17(-0.57,0.91)$ & $0.46(97.4)$ & 0.648 & $-0.2(-0.94,0.54)$ & $-0.54(97.4)$ & 0.594 \\
\hline IQ & WASI & $0.52(0.1,0.94)$ & $2.48(75.7)$ & 0.015 & $0.71(0.29,1.13)$ & $3.39(75.7)$ & 0.001 \\
\hline Verbal fluency & FAS & $0.14(-0.21,0.48)$ & $0.80(83.1)$ & 0.428 & $0.52(0.17,0.86)$ & $2.99(83.1)$ & 0.004 \\
\hline Perceived deficits & PDQ & $0.06(-0.44,0.57)$ & $0.24(70.8)$ & 0.811 & $0.18(-0.33,0.68)$ & $0.71(70.8)$ & 0.483 \\
\hline $\begin{array}{l}\text { Psychosocial } \\
\text { functioning }\end{array}$ & FAST & $-0.45(-0.76,-0.15)$ & $-2.95(65.1)$ & 0.004 & $-0.49(-0.8,-0.18)$ & $-3.19(65.1)$ & 0.002 \\
\hline Goal attainment & GAS & $3.46(2.33,4.58)$ & $6.14(61.2)$ & $<0.001$ & $2.02(0.89,3.14)$ & $3.58(61.2)$ & 0.001 \\
\hline
\end{tabular}

Abbreviations: Cl, confidence interval; $d f$, degrees of freedom; DS, Digit Span; DSST, Digit Symbol Substitution Test; FAS, verbal fluency test; FAST, Functioning Assessment Short Test; GAS, goal attainment scale; PDQ, perceived deficits questionnaire; SES, standardised effect size; SS, Symbol Search; UPSA, UCSD Performance-based Skills Assessment; VPA1, Verbal Paired Associates I; WASI, IQ.

\section{5 | DISCUSSION}

These results indicate high feasibility and acceptability of individual, therapist-led CRT using the established CIRCuiTS programme, as a potential treatment to enhance cognition and functioning for $B D$, with promising effect sizes on cognitive and functional outcomes.

\section{1 | Feasibility and acceptability}

The ADePT (Decision-making after Pilot and feasibility Trials) process aims to stimulate systematic identification and consideration of problems and solutions arising from feasibility trials and enhance the decision-making process in progressing to future studies. ${ }^{33}$ While a priori progression criteria appear optimal, ascertaining the potential for progression post-hoc is often more appropriate to maximise comparability between the present trial findings and the most relevant, high-quality research published to date. For example, cognitive trial recommendations ${ }^{31}$ and the only randomised individual CRT trial for $\mathrm{BD}^{9}$ were not available by the time of $\mathrm{CRiB}$ protocol finalisation. ADePT considers issues related to whether it is feasible to achieve adequate power in a future efficacy trial based on sample size calculations using effect sizes indicated from pilot trial outcomes: We report mostly medium ES in line with previous work. ${ }^{9}$ Achieving adequate power also relies on the feasibility of meeting recruitment targets by identifying sufficient participants who are eligible and willing to consent (in $\mathrm{CRiB}$, similar to previous trials ${ }^{9,10,15}$ ), the number of patients engaged with intervention and completing the trial (CRiB being equivalent or better compared to previous similar studies ${ }^{9,14,34}$ with its $12 \%$ loss to follow-up rate and high therapy completion rate) between the number of required sites (multisite differences not assessed in the single-site CRiB study). In addition, we did not experience issues with key aspects of the trial design (randomisation, blinding) or cost and duration of intervention (with health economic analyses indicating non-significant service use/QALY differences between CRT and TAU groups). Outcome selection was also in accordance with taskforce recommendations ${ }^{31}$; thus taken together, our findings suggest an efficacy trial of CRT for BD is both feasible and justified in terms of its putative benefits to patients.

The CRT satisfaction questionnaire indicated high acceptability, which we aim to examine more comprehensively in qualitative analyses for understanding and potentially refining the CIRCuiTS treatment manual tailored to people with BD. Five non-completers' participation deviated from the trial protocol (one due to ineligibility, four whose therapist did not adhere to protocols). Sensitivity analyses removing these participants indicate somewhat higher ES after they were removed. The protocol deviations alone suggest that patients with active substance-use disorders should be advised to seek help for this before undertaking CRT and emphasises the need to prioritise therapist adherence to protocols when designing future trials (ie, carefully consider therapist selection to ensure their adherence to study and treatment protocols, and engagement with participants). After excluding the protocol-deviation participants, the therapy completion rate was $93 \%$ (7\% study withdrawals due to illness or life events) which indicates very high feasibility of CIRCuiTS. Minimal adverse events also imply safety. Future efficacy studies should examine the long-term cost-effectiveness of CIRCuiTS vs TAU in the months and years following therapy completion, as is 


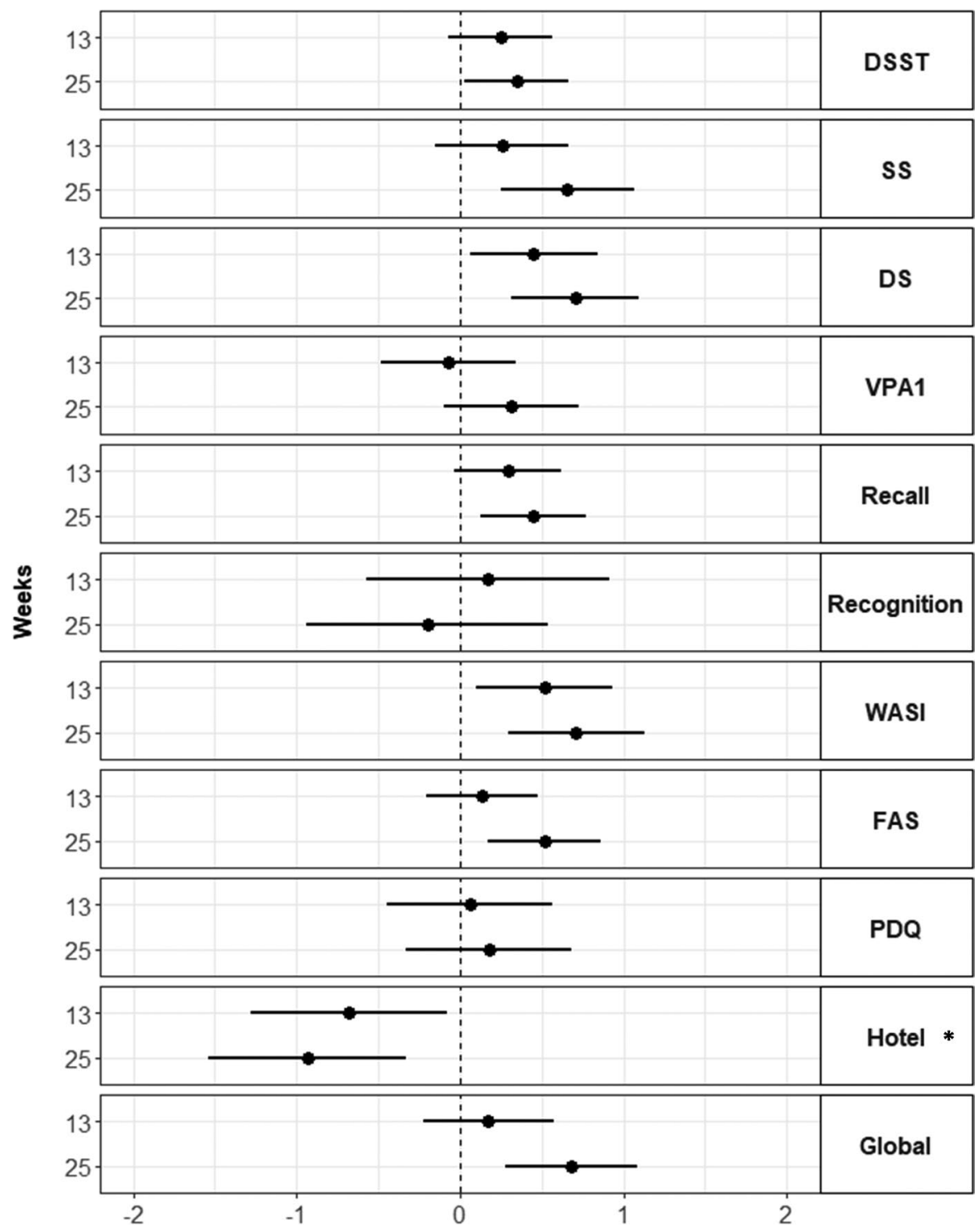

FIGURE 2 cognitive outcomes after CRT and TAU. Effect size (time $x$ group interactions) at week 13 and week 25 compared to pretreatment cognitive performance. ${ }^{*}$ The Hotel test was reverse-scored, meaning that a negative effect size represents greater improvement. Abbreviations: DSST = Digit Symbol Substitution Test (processing speed and attention), SS = Symbol Search (processing speed and attention), DS = Digit Span (working memory), VPA1 = Verbal Paired Associates (verbal learning), WASI = IQ, FAS = verbal fluency test, $\mathrm{PDQ}=$ perceived deficits questionnaire (subjective deficits), Hotel = hotel test of executive function, Global = composite measure of cognitive function

being undertaken for individuals with psychosis ${ }^{35}$; if inpatient admissions are reduced this would certainly indicate promising costeffectiveness of the intervention.

\section{2 | Putative effects of CRT}

Despite a relatively small sample size, large effects on cognition and functioning were observed, enduring for three months after the end of therapy. These are similar to effects observed in CRT for schizophrenia ${ }^{5,6}$ and equivalent ${ }^{9}$ or greater than ${ }^{10,34}$ previous $\mathrm{BD}$ trials. As reported in recent systematic reviews ${ }^{3,4}$ a handful of studies investigating related interventions for BD.
A distinct intervention focusing on functional rather than cognitive remediation has been supported in a randomised trial finding small benefits in functioning compared to psychoeducation treatment. ${ }^{34}$ Specific CRT programmes have been tested in 4 studies. Initially, one open-label study reported cognitive and clinical improvements particularly for participants less cognitively impaired at baseline $(\mathrm{N}=18) .{ }^{36}$ Conclusions are limited by the single-arm study design but this promoted CRT's potential for this population. Two subsequent group-CRT studies followed; one naturalistic study reporting non-significant cognitive benefits $(N=26)$ 37 and a randomised study finding benefits to subjective but not objective cognitive tests. ${ }^{10}$ The group CRT studies conclude that more intensive, therapist-led individual CRT emphasizing 
metacognitive skills and strategy use would likely yield meaningful (cognitive and functional) benefits. These were key elements of the CIRCuiTS therapy in this trial and the larger effects that we report compared to previous BD studies may be attributable to this. This speculation is supported further by the reports from the only other randomised trial examining individual CRT for individuals with $B D$, whose CRT intervention consisted of intensive training with multiple weekly sessions over 24 weeks. ${ }^{9}$ This randomised trial was for patients with psychotic bipolar disorder, representing a slightly different population to $\mathrm{CRiB}$, but reported similar medium/high effect sizes to those we present here. ${ }^{9}$ Together, the extant studies support the use of time-intensive individual cognitive remediation for people with BD but a focus is needed on therapy components in upcoming studies.

Some cognitive improvements were not evident in our study; notably the participants' perceived deficits, which contrasts somewhat with CRT satisfaction responses and objective performance tests, but possibly reflects an increased awareness of individuals' own cognitive weaknesses after CRT (especially given the metacognitive focus of the intervention). Other CRT programmes trialled for BD have not emphasised metacognitive skill development and have either not assessed subjective cognitive impairments ${ }^{9}$ or reported subjective improvements in the absence of objective cognitive improvements after CRT. ${ }^{10}$ In the original study protocol, the putative primary outcome was the DSST test of processing speed and attention due to its reported sensitivity to change. At follow-up, this was significantly improved by CRT, with similarly large ES observed in working memory, IQ and executive function. The IQ improvement, although a validated two-subtest measure, may have been driven by the matrix reasoning rather than vocabulary subtest in the WASI; matrix reasoning is a visual problem solving task which could reflect executive functions and be more susceptible to change than vocabulary domain. It may be that CRT targets on executive function are particularly vital for transfer of gains from cognition to everyday functioning, as has been found for people with schizophrenia. ${ }^{38}$ Goal attainment yielded the largest effect size, whose size may be attributable to participants creating personally meaningful cognitive and functional goals that would have been directly and explicitly targeted in therapy. However, assessments of functioning are likely to be associated with cognition: both the FAST (which includes a domain of cognitive functioning in everyday situations) and the GAS (where participants' goals may relate to cognitive functioning). Inter-correlations between individual cognitive tests should also be considered, as in all neuropsychological batteries, due to measurement of overlapping domains.

\subsection{The influence of pre-existing cognitive impairment}

For populations with psychosis, CRT benefits appear more pronounced for individuals with more extensive pre-existing cognitive impairments and it is widely recommended to recruit only participants with existing deficits. For individuals with BD this is not supported by the existing literature and enhancing cognition appears to be beneficial in terms of quality-of-life even for those without cognitive scores below demographically-corrected normative values. This is supported by current findings; at baseline, over one third of the CRiB sample scored $>0.5 \mathrm{SD}$ below the mean global composite measure and two thirds scored low on at least two individual cognitive tests, which is comparable to previous literature focusing on rates of cognitive impairment in those with BD. ${ }^{2}$ The CRiB sample size is insufficient to examine whether existing cognitive impairments affected subsequent improvement; future trials should assess whether CRT is recommended for those without deficits. The same is true when considering functional impairment; the data reported here indicate that the participants recruited in the CRiB study were representative in terms of cognition and functioning compared to other published samples (with 55\% showing moderate or severe functional impairment, in comparison with $51 \%$ previously reported using these FAST severity cut-offs). ${ }^{32}$

\section{4 | Strengths and limitations}

Notable strengths of this study include trial preregistration, a low proportion of missing data, the use of intention-to-treat analyses, stringent randomisation, and blinding of assessment researchers. The ability to determine benefits of CRT are limited by the feasibility nature of the study and correspondingly small sample size. The sample size prevented a more in-depth examination of the effects of CRT; we were unable to adjust for factors known to influence cognition such as various medications (psychotropic and somatic), physical illness, and sleep quality. We stratified randomisation based on the intensity of usual care patients received at baseline, which balanced TAU between groups; however, there is at present little justification for taking this decision in a future trial and stratifying based on cognitive impairment would likely be more beneficial, not least for ensuring that baseline impairment is well balanced between randomisation allocations. In this trial, CRT/TAU groups showed little difference on cognitive or functional variables except that verbal recall performance was better in the CRT group at baseline (ES $=0.6$ and was one of the few domains which did not show a relative improvement in CRT compared with TAU participants at follow-up). This occurrence can happen in small trials and is addressed through adjusting for baseline performance in linear mixed models, but one would expect poor performance to precede higher effect sizes based on the notion of "room for improvement." ${ }^{31}$ This is an assumption made in recommendations for trials to recruit only cognitively impaired individuals; namely the ISBD taskforce recommendations which were not available at the time of CRiB initiation; however, more evidence to support or refute this is certainly warranted. It is also worth noting that cognitive assessments (and the UPSA) have known practise effects which were addressed through between-group comparisons 
and baseline adjustment in linear mixed models. The results of this $\mathrm{RCT}$ require replication in larger, more definitive trials with multiple sites (since single studies such as CRiB limit generalisability of findings). Our participants were predominantly white, British and educated; more diverse samples are needed in future trials to better represent $\mathrm{BD}$ populations and improve generalisability of results. We recommend that these trials also seek to establish the core therapeutic components of CRT, the role of metacognitive skill development and whether there is a "target" group of patients with BD who would confer the most meaningful and enduring benefits from CRT, which would obviate unnecessary resource-intensive studies. In the current study, outcome assessors were instructed to disclose any incidents of inadvertent unblinding and an alternative assessor undertook these outcome assessments. Because procedures for recording and addressing incidents of unblinding were not formalised, it cannot be confirmed whether any assessments were undertaken by assessors who might have suspected which group the participant had been allocated to. Future trials should therefore formally assess whether, despite blinding, inadvertent beliefs about which trial arm participants were allocated could influence trial outcomes.

\section{5 | Future implications}

While current clinical practice commonly uses pharmacological treatments targeting acute affective symptoms, clinical evidence ${ }^{39}$ and our engagement work indicates that service users desire additional psychological therapies which may enhance quality-of-life more broadly. CIRCuiTS may be a good candidate to address these needs. Previous examination of CRT for bipolar disorder is limited and reports mixed findings, often from non-randomising studies or using less evidencebased programmes. ${ }^{1}$ The CRiB study sought to fill this evidence gap in setting a precedent for future work to establish whether this intervention has real potential for people with BD via efficacy and mechanistic trials. Considering the present findings, we urge future CRT studies to use robust methodology and manualised interventions to advance comprehension of the putative benefits of CRT and accumulate goodquality evidence to inform clinical practice.

\section{ACKNOWLEDGEMENTS}

This research was funded by the National Institute of Health Research (NIHR) Research for Patient Benefit program (ID PB-PG0614-34075) and NIHR Maudsley Biomedical Research Centre. This article presents independent research; the funder did not have a role in the design and conduct of the study; collection, management, analysis, and interpretation of the data; preparation, review, or approval of the manuscript; or the decision to submit the manuscript for publication. The views expressed are those of the authors and not necessarily those of the NHS, the NIHR or the Department of Health. The authors thank the study participants, our service user representatives, the CIRCuiTs program developers, support and trainers, the King's College Hospital Clinical Research Facility (CRF), the NIHR FAST-R service, King's College Clinical Trials Unit,
OPTIMA mood disorders service, SLaM Affective Disorders Service and the SLaM Consent for Contact (C4C) system. The authors thank the other individuals who contributed to the CRiB study, including students and researchers from the Centre for Affective Disorders (VN, EO, AJC, DS, PS, JS, RZ), psychiatrists who referred patients (MIH, SP, CB), the LQD study team (LM, RT, VA, EI), student placement helpers (SP, CG, MC, RIS).

\section{ORCID}

Rebecca Strawbridge (iD https://orcid.org/0000-0002-2984-1124

Dimosthenis Tsapekos (iD https://orcid.org/0000-0002-1972-4813 John Hodsoll iD https://orcid.org/0000-0001-7546-9901

Tim Mantingh http://orcid.org/0000-0002-3414-6388

Nefize Yalin (iD https://orcid.org/0000-0003-4298-5074

Paul McCrone iD https://orcid.org/0000-0001-7001-4502

Janet Boadu (iD https://orcid.org/0000-0001-7012-3514

Karine Macritchie (iD https://orcid.org/0000-0003-3178-359X

Matteo Cella iD https://orcid.org/0000-0002-5701-0336

Clare Reeder (iD https://orcid.org/0000-0001-9151-1946

Jessica Fish (iD https://orcid.org/0000-0002-9337-1489

Til Wykes iD https://orcid.org/0000-0002-5881-8003

Allan H. Young http://orcid.org/0000-0003-2291-6952

\section{REFERENCES}

1. Miskowiak KW, Carvalho AF, Vieta E, Kessing LV, Kessing LV. Cognitive enhancement treatments for bipolar disorder: A systematic review and methodological recommendations. Eur Neuropsychopharmacol. 2016;26(10):1541-1561.

2. Burdick KE, Russo M, Frangou S, et al. Empirical evidence for discrete neurocognitive subgroups in bipolar disorder: clinical implications. Psychol Med. 2014;44(14):3083-3096.

3. Bellani M, Biagianti B, Zovetti N, et al. The effects of cognitive remediation on cognitive abilities and real-world functioning among people with bipolar disorder: A systematic review. J Affect Disord. 2019; 257:691-697.

4. Tsapekos D, Seccomandi B, Mantingh T, et al. Cognitive enhancement interventions for people with bipolar disorder: a systematic review of methodological quality, treatment approaches, and outcomes. Bipolar Disord. 2020;22(3):216-230.

5. Wykes T, Huddy V, Cellard C, et al. A meta-analysis of cognitive remediation for schizophrenia: methodology and effect sizes. Am J Psychiatry. 2011;168(5):472-485.

6. Anaya C, Martinez Aran A, Ayuso-Mateos JL, et al. A systematic review of cognitive remediation for schizo-affective and affective disorders. J Affect Disord. 2012;142(1-3):13-21.

7. Reeder C, Huddy V, Cella $M$, et al. A new generation computerised metacognitive cognitive remediation programme for schizophrenia (CIRCuiTS): a randomised controlled trial. Psychol Med. 2017;47(15):2720-2730.

8. Trotta A, Murray RM, MacCabe JH. Do premorbid and post-onset cognitive functioning differ between schizophrenia and bipolar disorder? A systematic review and meta-analysis. Psychol Med. 2015;45(2):381-394

9. Lewandowski KE, Sperry SH, Cohen BM, et al. Treatment to enhance cognition in bipolar disorder (TREC-BD): Efficacy of a randomized controlled trial of cognitive remediation versus active control. J Clin Psychiatry. 2017;78(9):e1242-e1249.

10. Demant KM, Vinberg $M$, Kessing LV, Miskowiak KW. Effects of short-term cognitive remediation on cognitive 
dysfunction in partially or fully remitted individuals with bipolar disorder: Results of a randomised controlled trial. PLoS One. 2015;10(6):e0127955.

11. Strawbridge R, Fish J, Halari R, et al. The Cognitive Remediation in Bipolar (CRiB) pilot study: study protocol for a randomised controlled trial. Trials. 2016;29(17):371.

12. Hamilton M. A rating scale for depression. J Neurol Neurosurg Psychiatry. 1960;23:56-62.

13. Young RC, Biggs JT, Ziegler VE, Meyer DA. A rating scale for mania: reliability, validity and sensitivity. $\mathrm{Br} J$ Psychiatry. 1978;133:429-435.

14. Chisholm D, Knapp MRJ, Knudsen HC, et al. Socio-Demographic and Service Receipt Inventory - European Version: development of an instrument for international research: EPSILON Study 5. Br J Psychiatry. 2000;177(S39):s28-33.

15. Drake RJ, Day CJ, Picucci R, et al. A naturalistic, randomized, controlled trial combining cognitive remediation with cognitive-behavioural therapy after first-episode non-affective psychosis. Psychol Med. 2014;44(9):1889-1899.

16. Reeder C, Pile V, Crawford P, et al. The feasibility and acceptability to service users of CIRCuiTS, a computerized cognitive remediation therapy programme for schizophrenia. Behav Cogn Psychother. 2016;44(3):288-305.

17. Rose $D$, Wykes $T$, Farrier $D$, et al. What do clients think of cognitive remediation therapy? A consumer-led investigation of satisfaction and side effects. Am J Psychiatr Rehabil. 2008;11(2):181-204.

18. Dolan P. The measurement of health-related quality of life for use in resource allocation decisions in health care. In: Handbook of Health Economics. Amsterdam, Netherlands: Elsevier, 2000:1723-1760.

19. Wechsler WD. Adult Intelligence Scale-Fourth Edition. London, UK: Pearson; 2010.

20. Wechsler D. Wechsler Memory Scale-Fourth Edition (WMS-IV) Technical and Interpretive Manual. London, UK: Pearson; 2009.

21. Wechsler D. Wechsler Abbreviated Scale of Intelligence, 2nd edn., London, UK: Pearson; 2011.

22. Delis D, Kaplan E, Kramer J. Delis-Kaplan executive function system (DKEFS). San Antonio, TX: Psychological Corporation; 2001.

23. Manly T, Hawkins K, Evans J, et al. Rehabilitation of executive function: facilitation of effective goal management on complex tasks using periodic auditory alerts. Neuropsychologia. 2002;40(3): 271-281.

24. Sullivan MJ, Edgley K, Dehoux E. A survey of multiple sclerosis: I. Perceived cognitive problems and compensatory strategy use. Canadian. J Rehabil. 1990;4(2):99-105.

25. Patterson TL, Goldman S, McKibbin CL, et al. UCSD PerformanceBased Skills Assessment: development of a new measure of everyday functioning for severely mentally ill adults. Schizophr Bull. 2001;27(2):235-245.

26. Rosa AR, Sánchez-Moreno J, Martínez-Aran A, et al. Validity and reliability of the Functioning Assessment Short Test (FAST) in bipolar disorder. Clin Pract Epidemiol Ment Health. 2007;3(1):5.

27. Turner-Stokes L. Goal attainment scaling (GAS) in rehabilitation: a practical guide. Clin Rehabil. 2009;23(4):362-370.

28. Browne RH. On the use of a pilot sample for sample size determination. Stat Med. 1995;14(17):1933-1940.
29. Core R, Team R. A language and environment for statistical computing. R package 3.6.1. Vienna, Austria, 2019http://www.R-proje ct.org/

30. Curtis LA, Burns A. Unit Costs of Health and Social Care. 2015. http://www.pssru.ac.uk/project-pages/unit-costs/2015/index. php

31. Miskowiak KW, Burdick KE, Martinez-Aran A, et al. Methodological recommendations for cognition trials in bipolar disorder by the International Society for Bipolar Disorders Targeting Cognition Task Force. Bipolar Disord. 2017;19(8):614-626.

32. Bonnín CM, Martínez-Arán A, Reinares M, et al. Thresholds for severity, remission and recovery using the functioning assessment short test (FAST) in bipolar disorder. J Affect Disord. 2018;240:57-62.

33. Bugge $C$, Williams $B$, Hagen $S$, et al. A process for Decision-making after Pilot and feasibility Trials (ADePT): development following a feasibility study of a complex intervention for pelvic organ prolapse. Trials. 2013;14:353.

34. Torrent C, del Bonnin CM, Martínez-Arán A, et al. Efficacy of functional remediation in bipolar disorder: A multicenter randomized controlled study. Am J Psychiatry. 2013;170(8):852-859.

35. Wykes T, Joyce E, Velikonja T, et al. The CIRCuiTS study (Implementation of cognitive remediation in early intervention services): protocol for a randomised controlled trial. Trials. 2018;19:183.

36. Deckersbach $T$, Nierenberg AA, Kessler R, et al. Cognitive rehabilitation for bipolar disorder: An open trial for employed patients with residual depressive symptoms. CNS Neurosci Ther. 2010;16(5):298-307.

37. Veeh J, Kopf J, Kittel-Schneider S, et al. Cognitive remediation for bipolar patients with objective cognitive impairment: A naturalistic study. Int. J Bipolar Disord. 2017;5(1):8. https://doi.org/10.1186/ s40345-017-0079-3

38. Wykes T, Reeder C, Huddy V, et al. Developing models of how cognitive improvements change functioning: Mediation, moderation and moderated mediation. Schizophr Res. 2012;138(1): 88-93.

39. Nestsiarovich A, Hurwitz NG, Nelson SJ, et al. Systemic challenges in bipolar disorder management: A patient-centered approach. Bipolar Disord. 2017;19(8):676-688.

\section{SUPPORTING INFORMATION}

Additional supporting information may be found online in the Supporting Information section.

How to cite this article: Strawbridge R, Tsapekos D, Hodsoll $J$, et al. Cognitive remediation therapy for patients with bipolar disorder: A randomised proof-of-concept trial. Bipolar Disord. 2021;23:196-208. https://doi.org/10.1111/bdi.12968 